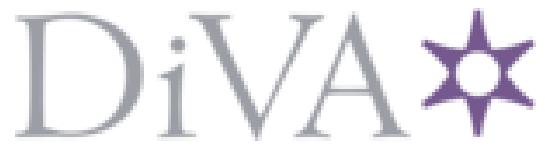

http://www.diva-portal.org

Preprint

This is the submitted version of a chapter published in Hajnal Andréka and István Németi on Unity of Science: From Computing to Relativity Theory Through Algebraic Logic.

Citation for the original published chapter:

Westerståhl, D., van Benthem, J. (2021)

Generalized Quantifiers Meet Modal Neighborhood Semantics

In: Judit Madarász, Gergely Székely (ed.), Hajnal Andréka and István Németi on Unity of Science: From Computing to Relativity Theory Through Algebraic Logic Springer Outstanding Contributions to Logic

N.B. When citing this work, cite the original published chapter.

Permanent link to this version:

http://urn.kb.se/resolve?urn=urn:nbn:se:su:diva-191598 


\title{
Generalized Quantifiers Meet Modal Neighborhood Semantics
}

\author{
Johan van Benthem \& Dag Westerståhl
}

April 20, 2019

We dedicate this paper to Hajnal and István, in friendship and admiration.

\section{Introduction: quantifiers and neighborhoods}

Hajnal Andréka and István Németi have long been leaders in the algebraic study of the foundations of logic. In particular, the high abstraction levels provided by their expertise in algebra, with a judicious influx of model-theoretic ideas from modal logic, have led to new perspectives on well-established core systems of the field such as first-order predicate logic. A case in point are the decidable guarded fragment and the generalized first-order semantics found in Andréka et al. (1998). In a recent paper Andréka et al. (2017), triggered by work of Aldo Antonelli on weak first-order logics, this abstraction was taken one step further, viewing existential and universal quantifiers as arbitrary generalized quantifiers that can be parametrized to objects or tuples of objects in a model.

The authors of the latter paper suggest that this generalized quantifier perspective can be fruitfully compared to neighborhood semantics in modal logic, a generalization of the standard modal semantics with accessibility relations $R s t$ between points, that is finding ever more uses. A neighborhood model is a structure $\mathcal{M}=(W, N, V)$ with $W$ a non-empty set of worlds, $V$ a valuation mapping proposition letters to subsets of $W$, while the neighborhood relation $N s X$ is a binary point-to-set relation connecting each point $s$ to its neighborhoods $X$. Thus, a neighborhood relation is exactly the same as a point-parametrized unary generalized quantifier, in a precise sense to be explained below.

In this small piece, we explore this suggested analogy a little bit further by comparing three topics at the interface of generalized quantifier theory in linguistics and modal neighborhood logic. The attraction in pursuing this interface is not just technical, but also involves a meeting of different cultures. Intuitions about modal logic are often epistemic, temporal, or computational (van Benthem and Blackburn, 2007), whereas many of the intuitions underlying generalized quantifier theory involve connections with appealing empirical observations about natural language (Peters and Westerståhl, 2006). 
Incidentally, the above structural connection can be viewed in two ways. As described so far, a neighborhood frame $(W, N)$ has the type

$$
\langle e,\langle\langle e, t\rangle, t\rangle\rangle
$$

where $e$ is the type of entities, $t$ of truth values, and $\langle a, b\rangle$ of all functions from $a$-type entities to $b$-type ones. That is, $N$ is a relation between entities and sets of entities, or, equivalently, a function from entities to sets of sets of entities (its family of 'neighborhoods'). Next, a unary generalized quantifier on a domain $W$ is simply a sets of subsets of $W$. Thus, it is exactly what we will call a uniform neighborhood frame, meaning that $N$ is a constant function. Ordinary neighborhood frames are then point-parameterized unary generalized quantifiers. ${ }^{1}$ Now, this type is isomorphic to another, viz.: ${ }^{2}$

$$
\langle\langle e, t\rangle,\langle e, t\rangle\rangle
$$

This shift to a new, though equivalent, type makes our quantifiers (pointparameterized or not) into functions on the power set of the domain of primitive entities: shifting $(W, N)$ to $(W, F)$, where

$$
F(X)=\{s \in W: N s, X\}
$$

Taking this functional setting would bring our study closer to algebraic semantics for modal logics, involving a Boolean algebra with additional operators about which less or more can be assumed (monotonicity, intersection closure, and other special properties are well-known extras that will return later on in this article), cf. Blackburn et al. (2001); Venema (2006) for details and references on modal algebra. We will shift to the functional perspective occasionally in what follows, but the relational one will be our main vehicle.

The three topics that we will highlight in this paper concern basic features of the two realms to be connected. The first topic is the well-known locality of modal logics, where evaluation takes place in a local environment of the current point, viewed in tandem with the equally well-known conservativity of quantifiers in natural language, that come with restrictions to relevant subdomains of a whole model. The second topic is the role of fundamental invariance relations in both fields that constrain permissible denotations: notions of bisimulation in modal logic, and of permutation or isomorphism invariance for quantifiers. Our third topic concerns the core business of much of modal logic: finding new complete axiomatic systems for specific generalized quantifiers, now taking inspiration from generalized quantifier theory. In each case, we show how to make the comparison, point out which analogies are fruitful and which ones less so, we state a number of new observations, referring to current literature for deeper developments, and we end with an assessment and further ways to go.

\footnotetext{
${ }^{1}$ Admittedly, point-parameterized generalized quantifiers have not been studied much in the GQ literature, and in this paper we mostly restrict attention to standard generalized quantifiers, i.e. to uniform neighborhood frames.

${ }^{2}$ This equivalence can be derived formally in categorical logics such as the Lambek Calculus.
} 
In all of this, our main emphasis is on raising new kinds of questions, though we back them up by some new observations throughout.

We assume that the reader knows the basics of both fields to be connected. For a survey of generalized quantifier theory in logic and linguistics, we refer to Peters and Westerståhl (2006), and for an up-to-date introduction to modal neighborhood semantics, to the recent textbook Pacuit (2017).

\section{Locality and conservativity}

We start by recalling a few basic notions. The language of modal propositional logic has formulas constructed using proposition letters, Boolean connectives, and modal operators $\square, \diamond$. In relational models $\mathcal{M}=(W, R, V)$, a formula $\square \varphi$ is true at point $s$ (written $\mathcal{M}, s=\varphi$ ) if $\varphi$ is true at all $t$ with $R s t$ - and $\diamond \varphi$ is the existential dual. The same modal language can also be interpreted over neighborhood models $\mathcal{M}=(W, N, V)$, where $\square \varphi$ is now true at point $s$ if $N s,\{t \in W \mid \mathcal{M}, t \models \varphi\}$. In this case, we speak of modal neighborhood logic whose semantics also has an alternative 'monotone' version, explained below.

\subsection{Locality in modal semantics}

Standard modal logic satisfies locality, which is often taken to be a characteristic property of modal languages in general, cf. Blackburn et al. (2001). Technically, evaluating a modal formula $\varphi$ at a point $s$ in a model $\mathcal{M}=(W, R, V)$ yields the same truth value as evaluating $\varphi$ at $s$ in any submodel of $\mathcal{M}$ that contains $s$ and is closed under taking $R$-successors. This locality property extends to generalized topological semantics (van Benthem and Bezhanishvili, 2007): a modal formula $\varphi$ is true at $s$ in $\mathcal{M}$ iff $\varphi$ is true at $s$ in any restriction $\mathcal{M} \mid O$ of the model $\mathcal{M}$ to an open set $O$ containing $s$. Here a topological frame is a neighborhood frame $(W, N)$ where, for some topology on $W$, we have $N s, X$ iff for some open set $Y, s \in Y \subseteq X .^{3}$

Now both facts are instances of a more general observation in Bonnay and Westerståhl (2018). First, we need some definitions. Let $\mathcal{M}=(W, N, V)$ be a neighborhood model, and let $A$ be a subset of $W$. The submodel

$$
\mathcal{M} \mid A=\left(A, N_{A}, V_{A}\right)
$$

arises by restricting the domain of $\mathcal{M}$ to $A$, letting the new neighborhoods for a point $s$ be $\{X \cap A: N s, X\}$, cf. van Benthem and Pacuit (2011).

Modal formulas need not preserve their truth values in passing to arbitrary submodels, but there exist special submodels where they do. A generated submodel of $\mathcal{M}$ is a submodel $\mathcal{M} \mid A$ satisfying the following equivalence:

$$
\text { for all } s \in A \text { and all } X, N s, X \text { iff } N_{A} s, X \cap A
$$

\footnotetext{
${ }^{3}$ In the above-mentioned functional version of neighborhood models, a topological frame would be of the form $(W, F)$, where $F$ is the interior function of the topology.
} 


\section{Fact 1}

If $\mathcal{M} \mid A$ is a generated submodel of $\mathcal{M}$, then for all $s \in A$ and all formulas $\varphi$, $\mathcal{M}, s \models \varphi$ iff $\mathcal{M} \mid A, s \models \varphi$.

Proof. By a straightforward induction on modal formulas. To show how the above definition works, here is the inductive case for the modal operator $\square \psi$. We let $\llbracket \varphi \rrbracket_{\mathcal{M}}=\{s \in W: \mathcal{M}, s \models \varphi\}$. For $s \in A$, we have the following equivalences:

$$
\begin{array}{rlll}
\mathcal{M} \mid A, s \models \square \psi & \text { iff } & N_{A} s, \llbracket \psi \rrbracket_{\mathcal{M} \mid A} & \\
\text { iff } & N_{A} s, \llbracket \psi \rrbracket_{\mathcal{M}} \cap A & \text { (induction hypothesis) } \\
\text { iff } & N s, \llbracket \psi \rrbracket_{\mathcal{M}} & \text { (generated submodel) } \\
\text { iff } & \mathcal{M}, s \models \square \psi . &
\end{array}
$$

Now, it is easy to see that in the case of the relational semantics, the above notion of generated submodel is exactly the usual one. Likewise, in the topological semantics, the subsets which yield generated submodels are precisely the open sets. Thus, these two are special instances of our Fact.

It is time to highlight a distinction between two versions of the semantics for modal neighborhood logic. The proof above uses the precise semantics, where

$$
\mathcal{M}, s \models \square \varphi \text { iff } N s, \llbracket \varphi \rrbracket_{\mathcal{M}}
$$

However, there is also a monotone semantics, with the truth condition

$$
\mathcal{M}, s \models^{m} \square \varphi \text { iff there is } X \text { such that } N s, X \text { and } X \subseteq \llbracket \varphi \rrbracket_{\mathcal{M}}^{m}
$$

In the topological semantics for modal logic, and in the standard relational semantics, the two versions are equivalent. But in general, when the families $\{X \subseteq W \mid N s, X\}$ need not be closed under supersets, $\models$ and $\models^{m}$ may differ. Interestingly, though, the distinction does not matter for the current topic:

\section{Fact 2}

Invariance for generated submodels holds under the monotone semantics as well.

Proof. An analogous inductive argument goes through for $\models^{m}$ too.

While the precise semantics is the absolute minimum needed for algebraic semantics, there are some technical complications in its theory (cf. Pacuit, 2017), while also, the monotone semantics is the one that occurs in most applications. Hence we will use the latter in the sequel, unless explicitly indicated otherwise.

In standard relational semantics, there is a smallest local environment for which the above invariance holds, namely, the smallest generated submodel whose domain consists of the point $s$ and all points reachable from it in a finite number of $R$-steps. This is no longer true in the topological semantics, where 
open sets can decrease without limit, and a fortiori, this uniqueness does not hold in neighborhood semantics. More precisely, given $(W, N)$ and $s \in W$, let

$$
A^{s}=\bigcap\left\{A \subseteq W: s \in A \text { and }\left(A, N_{A}\right) \text { is a generated subframe of }(W, N)\right\}
$$

If $\left(A^{s}, N_{A^{s}}\right)$ is itself a generated subframe of $(W, N)$, then it is the smallest such subframe containing $s$. But it need not be generated subframe of $(W, N)$ :

Counter-example. Consider the neighborhood frame $(\mathbb{N}, N)$, where for $n>0$, $N n, X$ holds iff $n \in X$, but $N 0, X$ holds iff $0 \in X$ and $X$ is co-finite. Since for all $k$, the set of $X \subseteq \mathbb{N}$ such that $N k, X$ holds is a filter, even the minimal normal modal logic $\mathrm{K}$ is sound for $(\mathbb{N}, N)$, but it is easily checked that $\left(A^{0}, N_{A^{0}}\right)$ is not a generated subframe according to the above definition.

Indeed, imposing the existence of smallest neighborhoods in topological semantics takes us back to relational semantics. This is the well-known sense in which relational models correspond to special topological 'Alexandrov spaces' (cf. van Benthem and Bezhanishvili, 2007). But here is a slightly different take in our current terms. In relational semantics, let $R_{s}$ denote the set of $R$-successors of $s$. The following fact can be found in Bonnay and Westerståhl (2018):

\section{Fact 3}

If $(W, N)$ is a topological neighborhood frame such that for all $s \in W,\left(A^{s}, N_{A^{s}}\right)$ is a generated subframe of $(W, N)$, then $(W, N)$ is in fact a relational frame, in the sense that there is a unique relation $R \subseteq W^{2}$ such that $N s, X$ iff $R_{s} \subseteq X$.

Thus, neighborhood modalities may have a variety of local environments in a model — and under special circumstances, even a smallest one.

\subsection{Conservativity and domain restriction for quantifiers}

A binary quantifier on a domain $W$ is a binary relation between subsets of $W$. These appear in English as the interpretations of determiner phrases, such as "every", "no", "most", "exactly four", "all but three", "more than two-thirds of the", and of adverbs like "always", "usually", "never". Other languages may use different linguistic constructions for these purposes, but it is a well-attested empirical fact that quantifiers across human languages all satisfy the following equivalence (cf. Peters and Westerståhl, 2006):

$$
Q A B \text { iff } Q A(A \cap B) \quad \text { Conservativity }
$$

The appeal of conservativity, and perhaps even an explanation of its ubiquity, stems from its role in facilitating efficient 'local' processsing: in understanding an expression, we can restrict attention to various smaller relevant subdomains. This processing restriction can also occur with unary generalized quantifiers $Q B$ (think of expressions like "everyone", "someone"), where $Q$ merely denotes some family of subsets of the whole domain of individuals. 
Now, this resembles what we saw for modal logic. Barwise and Cooper (1981) say that a unary quantifier $Q$ lives on a set $A$ (which can be taken to be a subset of the domain) if for all subsets $B$ of the domain, $Q B$ iff $Q B \cap A$. In these terms, a binary quantifier $Q^{\prime}$ is conservative iff for every subset $A$ of the domain, the restriction of $Q^{\prime}$ to $A$ (the unary quantifier obtained by fixing the first argument to $A$ ) lives on $A$. This is exactly the clause we used in defining generated submodels. The domains of such models are live-on sets.

But there is more to the comparison. In the modal case, the restriction to the local environment $A$ is drastic: we ignore $W-A$ and evaluate all formulas inside the submodel $\mathcal{M} \mid A$. Thus, we perform what in logic is called a relativization of the model. But this is not the intent of Conservativity. When we say

\section{All fans wore a cap,}

we are merely saying that all fans were fans who were cap wearers, not that all fans wore a cap that was itself a fan: the embedded quantifier "a cap" can look outside of the domain of fans. ${ }^{4}$

Even so, "a cap" will impose its own form of conservativity: both quantifiers impose restrictions on arguments of the transitive verb " $x$ wears $y$ ", where $x$ gets restricted to fans, and $y$ to caps. The analogue for modal logic might be iterated modalities referring to different points with different neighborhoods, where liveon sets can be different at different points. But of course, the basic modal language has no explicit syntactic counterpart for binary relational atoms. ${ }^{5}$

One way of highlighting the difference between the two frameworks focuses on the binary nature of linguistic quantifiers $Q A B$. Its immediate reflection in a modal neighborhood language would not be a unary modality $\square \varphi$ but rather a binary modality $\square \varphi \psi$ referring to a binary neighborhood relation $N s, X Y$ in a straightforward manner. Such binary modalities have been little used so far, though they occur with weak implicational logics, cf. de Jongh and Shirmohammadzadeh (2018). In an implicational setting, we often think of an antecedent $\varphi$ as restricting attention to the submodel (not necessarily generated) consisting of all points in the model satisfying $\varphi$, while the consequent $\psi$ is evaluated in that set by reference to the whole model. Indeed, several types of conditional satisfy Conservativity, such as classical entailment saying that $\psi$ is true in all $\varphi$-worlds, or counterfactual entailment saying that $\psi$ is true in the closest $\varphi$-worlds to the current one. ${ }^{6}$ But Conservativity can fail for more complex assertions. A temporal conditional $\varphi \Rightarrow \psi$ saying that after every instance of $\varphi$, there will be a later instance of $\psi$, supports no valid implication to $\varphi \Rightarrow(\varphi \wedge \psi)$.

Thus, it seems of interest to determine what the conservativity constraint means in a modal language, or indeed, any logical language. Can we tell from the syntactic logical form of a statement when this behavior occurs? We give two

\footnotetext{
${ }^{4}$ The difference between relativization and conditionalization is ubiquitous. For a discussion in the setting of dynamic-epistemic logics of model update, cf. van Benthem (2011).

${ }^{5}$ For non-iterated modalities, however, the relativizations of unary quantifiers are exactly the binary quantifiers satisfying Conservativity and the Extension property to be defined later.

${ }^{6}$ A generalized quantifier perspective on conditionals was proposed in van Benthem (1984).
} 
versions of such a result, which may be viewed as model-theoretic preservation theorems of a somewhat unusual kind.

First consider the language of propositional logic. Say that a formula $\varphi(p, q)$ with perhaps also other proposition letters beyond $p, q$, is $p$-conservative in $q$ if it satisfies the following validity:

$$
\models \varphi(p, q) \leftrightarrow \varphi(p, p \wedge q)
$$

\section{Fact 4}

A formula $\theta$ is $p$-conservative in $q$ iff it can be defined by a formula of the form $\varphi \vee(p \wedge \psi(q))$, where $\varphi$ contains no occurrences of $q$, and $p$ does not occur in $\psi$.

Proof. Obviously, formulas of this form are $p$-conservative in $q$. Conversely, if $\theta$ is $p$-conservative in $q$, consider any disjunctive normal form $\theta^{\prime}$ of $\theta$. Applying the valid equivalence $\theta^{\prime}(p, q) \leftrightarrow \theta^{\prime}(p, p \wedge q)$, we replace $q$ by $p \wedge q$ throughout. In this way, disjuncts where the literal $\neg p$ occurs together with $q$ become contradictions and can be dropped up to logical equivalence. Next, in disjuncts where the literal $\neg p$ occurs together with $\neg q$, the new conjunct $\neg(p \wedge q)$ can be dropped, since it already follows from $\neg p$, leaving no occurrence of $q$. Finally, all disjuncts in which $p$ appeared remain the same up to logical equivalence after the substitution. The resulting, still equivalent, formula is easily seen to be logically equivalent to a disjunction of a $q$-free formula plus a formula where we can put $p$ in front, followed by a $p$-free disjunction.

We can extend this style of analysis to richer logical languages. A very simple illustration, still allowing for the use of normal forms, is this. Say that a formula in monadic first-order logic is $P$-conservative in $Q$ if replacing occurrences of a subformula $Q x$ by $P x \wedge Q x$, for any variable $x$, results in an equivalent formula.

\section{Fact 5}

A formula $\theta$ in monadic first-order logic is $P$-conservative in $Q$ iff it is definable by a Boolean combination of (a) $Q$-free formulas, (b) formulas $\exists x(P x \wedge \psi)$ where $\psi$ is $P$-free and has no other free variables than $x$, and (c) for each variable $u$, a formula as in Fact 4 (with $p$ replaced by $P u, q$ by $Q u$, etc.).

Proof. Again, the direction from the special forms to Conservativity is obvious. In the other direction let, for simplicity, the relevant unary predicates be just $P, Q, R$. A 'state description of $x$ ' is a formula of the form $(\neg) P x \wedge(\neg) Q x \wedge$ $(\neg) R x . \theta$ is equivalent to a Boolean combination of atomic formulas $P x, Q y$, etc. and formulas of the form $\exists x s d(x)$, where $s d(x)$ is a state description of $x$; we can assume that the bound variable is the same in each. Treating all these as atoms, write the formula in disjunctive normal form; we obtain a formula $\theta^{\prime}$ equivalent to $\theta$ which is still $P$-conservative in $Q$. Now replace all occurrences of $Q u$, for all variables $u$, by $P u \wedge Q u$. In each disjunct of the normal form, we can apply the same argument as for Fact 4, both inside the quantified conjuncts and among conjuncts which are state descriptions $s d(u)$. In particular, inspecting the four 
relevant types of object: (a) $\exists x(P x \wedge Q x \wedge \ldots)$ and (b) $\exists x(P x \wedge \neg Q x \wedge \ldots)$ are unchanged up to logical equivalence after the substitution, (c) $\exists x(\neg P x \wedge Q x \wedge$ ...) becomes the contradictory $\exists x(\neg P x \wedge P x \wedge Q x \wedge \ldots)$ and can be dropped, and the final substitution (d) $\exists x(\neg P x \wedge \neg(P x \wedge Q x) \wedge \ldots)$ is equivalent to $\exists x(\neg P x \wedge \psi)$ with $\psi Q$-free, yielding a $Q$-free formula overall.

More challenging cases for determining the precise syntactic impact of conservativity would be the language of basic modal logic over relational models, or all of first-order logic. For the latter, we conjecture that it suffices to mark each occurrence of a quantifier by a unique bound variable, and then to make sure that each occurrence of an atom $Q x$ has its variable governed by a relativized quantifier $\exists x(P x \wedge \ldots)$ or $\forall x(P x \rightarrow \ldots)$. ${ }^{7}$ This syntactic class allows any $Q$-free formulas, and it is closed under all Booleans and quantifiers.

However this may be, our simple observations may already have shown how notions from generalized quantifier theory can generate new types of modeltheoretic question in the modal and first-order realm.

Coda and Caveat. Generalized Quantifier Theory also has other intuitions concerning locality. Let us view a quantifier $Q$ as a functor assigning to each model $\mathcal{M}$ a set $Q_{\mathcal{M}}$ of subsets of the domain. Then the following well-known constraint on how the functor $Q$ assigns its values says intuitively (in the binary case) that a quantifier $Q A B$ only cares about the two sets it is comparing:

\section{Extension}

For all models $\mathcal{M}, \mathcal{N}$ with $A \cup B$ included in both domains,

$$
Q_{\mathcal{M}} A B \text { iff } Q_{\mathcal{N}} A B
$$

Here we hit a difficulty in comparing the two sides of this paper, namely, generalized quantifier theory and neighborhood semantics for modal logic. The Extension constraint is harder to phrase in modal logic, or in logical systems in general. It requires a regimented class of models where the structure assigned to models is constrained. This is not the thinking in modal neighborhood semantics, where models can have arbitrary neighborhood relations. Instead, Extension says that, when models are related in some way (by the submodel relation, or perhaps another important cross-model relation), the quantifier structures in these models have to be similar. ${ }^{8}$ The intuition underlying this condition seems to be a coherence constraint: all models in the class are compatible, and are fragments of one big supermodel. This is not normally assumed in modal logic, where validity is a case-by-case notion. ${ }^{9}$

\footnotetext{
${ }^{7}$ In the modal case, without explicit variable binding, this description requires more care. The syntactic governing has to be done at the right level of modal operator depth, and there are further combinatorial complexities. For instance, the modal formula $\square(p \vee r) \wedge \square(q \vee r)$ is $p$-conservative in $q$ since it is equivalent (in the normal modal logic $\mathrm{K}$ ) to $\square(r \vee(p \wedge q))$.

${ }^{8}$ To find analogues for this in the modal realm, one might require, say, that accessibility relations should be the same in the overlap of any two models in one's model class.

${ }^{9}$ It might be of interest, however, to see what restrictions like this mean in the setting of modal logics for model-changing operations, cf. Aucher et al. (2018).
} 
It should be noted, however, that on the generalized quantifier side, Extension does have a clear syntactic counterpart. Say that a sentence $\varphi=\varphi(P, \ldots)$ is $P$-restricted if for all $\mathcal{M}, \mathcal{M}=\varphi \Leftrightarrow \mathcal{M} \mid P^{\mathcal{M}} \models \varphi$. This is stronger than $P$-conservativity: a $P$-restricted sentence is $P$-conservative in all other unary predicate symbols $Q$ occurring in it, not just a specific one. Now it is immediate that $\varphi$ is $P$-restricted iff it is equivalent to $\psi^{(P)}$ (the relativization of $\psi$ to $P$ ) for some $P$-free sentence $\psi$ (just replace $P x$ by $x=x$ in $\varphi$ and relativize).

We leave this topic open-ended here, though the mismatch will return with our next topic. A deeper connection may well have to be category-theoretic.

\section{Invariance and simulation}

\subsection{Modal logic and invariance}

Like many logical systems, modal logic allows for a semantic invariance analysis of its expressive power, (Blackburn et al., 2001). The basic notion of structural invariance that fits the expressive power of the modal language on neighborhood models comes in several varieties. We will couch the following discussion in terms of the monotone truth condition stated above for $\square \varphi$ :

Let $\mathcal{M}=(W, N, V)$ and $\mathcal{M}^{\prime}=\left(W^{\prime}, N^{\prime}, V^{\prime}\right)$. A binary relation $Z \subseteq W \times W^{\prime}$ is a bisimulation between $\mathcal{M}$ and $\mathcal{M}^{\prime}$ if (a) if $s Z t$, then $s \in V(p) \Leftrightarrow t \in V^{\prime}(p)$ for all atoms $p$, (b) if $s Z t$ and $N s, X$, then there is a set $Y$ with $N^{\prime} t, Y$ such that $\forall y \in Y \exists x \in X x Z y$; and vice versa starting from $s Z t$ and $N^{\prime} t, Y$.

\section{Fact 6}

Modal formulas are invariant for bisimulation.

The proof is by induction on modal formulas, where the inductive case for $\square \varphi$ mirrors exactly the back-and-forth clause of bisimulation. The fact explains our earlier observation about generated submodels. The identity is a bisimulation between the full model $\mathcal{M}$ and the generated submodel $\mathcal{M} \mid A$.

In logic, invariance is connected with definability and the genesis of language (van Benthem (2002)). Once we have invariant structure, the issue arises of languages expressively complete for defining it. ${ }^{10} \mathrm{~A}$ typical result in this vein is the theorem saying that the first-order formulas in a signature with binary $R$ and unary $P, Q, \ldots$ having one free variable $x$ that are invariant for bisimulation on relational models are precisely those definable in the basic modal language. For an exposition of this result and the literature around it, see Blackburn et al. (2001). The theorem was lifted to the neighborhood setting in Pauly (2001), using two-sorted first-order models with point and set objects, with an abstract neighborhood relation $N$ and membership relation $E .^{11}$

\footnotetext{
${ }^{10}$ Bisimulation is not the only structural relation between models for which this makes sense. One can also use isomorphism, potential isomorphism, or many other equivalence relations.

${ }^{11}$ Here we work in Henkin-style, without assuming that the set objects are a full power set.
} 


\section{Fact 7}

The formulas in a two-sorted first-order language for points and sets that are invariant for bisimulation are precisely those that are definable in the modal propositional language with the monotone neighborhood semantics.

Remark. The above result is not the only way of phrasing the issue. Given that bisimulation is also about preserving relational structure, one may ask for a richer notion of invariance with respect to relations, not just properties of points. For standard relational models, one such additional requirement is 'safety for bisimulation' (van Benthem, 1998), whose definition we forego here. The point is that, in such an extended language, we can now determine which definable operations on relations preserve bisimulation, and the answer for the first-order language is: the operations of regular algebra without iteration. Bisimulationsafe operations on neighborhood relations have been studied in Pauly (2001).

Remark. The above analysis extends to the precise neighborhood semantics for the modal language. In particular, the above notion of bisimulation can be modified to deal with the precise semantics for $\square \varphi$ defined earlier. But the solution involves several non-trivial subtleties: cf. Hansen et al. (2009), and the detailed explanations and references in Pacuit (2017). ${ }^{12} 13$

\subsection{Invariance and generalized quantifiers}

Prima facie, the same thinking applies to the realm of generalized quantifiers. There is a standard constraint on quantifiers which seems exactly in the same spirit, namely invariance for permutations or even isomorphism between models:

\section{Isomorphism Invariance}

If $F$ is a bijection between the domains of two models $\mathcal{M}$ and $\mathcal{N}$, then for all $A, B, Q_{\mathcal{M}} A B$ iff $Q_{\mathcal{N}} F[A] F[B]$.

In logic, isomorphism invariance is basic, but also permissive, many notions pass this test. So, what if we make the test stricter, replacing isomorphism by the rougher relation of bisimulation (cf. van Benthem and Bonnay (2008) for a systematic study)? Do we get a much narrower class of generalized quantifiers? Here is one way of formulating the constraint, where we lift the relation of bisimulation to a function between sets:

\section{Bisimulation Invariance}

If $Z$ is a bisimulation between the domains of models $\mathcal{M}, \mathcal{N}$, then for all sets $A, B \subseteq M$ and $C, D \subseteq N$,

$$
\text { (a) } Q_{\mathcal{M}} A B \text { iff } Q_{\mathcal{N}} Z[\bar{A}] Z[B] \text {, (b) } Q_{\mathcal{N}} C D \text { iff } Q_{\mathcal{M}} Z^{-1}[C] Z^{-1}[D] .{ }^{14}
$$

\footnotetext{
${ }^{12}$ The solution is not just a symmetric version of the above back-and-forth clause between the two matching neighborhoods. The latter rather fits the language of 'instantial neighborhood logic', van Benthem et al. (2017).

${ }^{13}$ For an analysis of a related challenge to bisimulation, cf. Baltag and Cinà (2018).

${ }^{14}$ We use the double clause since the maps $Z$ an $Z^{-1}$ induced by the relation $Z$ (i.e. $Z[A]=$ $\{c: \exists a \in A a Z c\}$ and $\left.Z^{-1}[C]=\{a: \exists c \in C a Z c\}\right)$ are not in general inverses.
} 
This condition seems plausible, and it suggests an elegant functional reformulation of the notion of bisimulation between neighborhood models. What it says is that the bisimulation relation $Z$, viewed as a function, and the neighborhood relation corresponding to a unary quantifier $Q$, viewed as a function from sets to sets by the type shift of Section 1, commute. The match is not precise, as will be clear by spelling out some details, but here is an intriguing point where we find a connection with co-algebra, cf. Hansen et al. (2009), Jacobs (2016). We do not pursue this line here, however, because there is also a problem.

Our aim was to classify generalized quantifiers as linguistic constructions in terms of invariance. But in the preceding scenario, the neighborhood relation corresponding to the quantifier is not a logical construction, but a relation among objects and individuals. Its invariance is the starting point, the same way in which 'structure-preserving' transformations of models take the invariance of the relevant atomic structure for granted. What one studies then is which further constructions over this base vocabulary define invariant notions.

The difference that gets in the way of a direct comparison is as with our discussion of Extension. The Isomorphism constraint assumes a functor assigning quantifiers to domains, a setting different from that of modal logic and model theory. ${ }^{15}$ Even with this tension, several interesting things can be asked about generalized quantifiers on the analogy with modal logic.

One issue is the earlier notion of safety. Given generalized quantifiers that satisfy some invariance across models, say, with respect to some notion of bisimulation, which operations on these quantifiers give rise to new defined quantifiers supporting invariance for that bisimulation? In this perspective, attention would shift to algebras of generalized quantifiers, on the analogy of algebras of neighborhood relations (cf. van Benthem, 2014).

A different, but also quite natural, way of connecting the modal and generalized quantifier perspectives runs via the extensive existing model theory of generalized quantifier languages such as $E L(Q)$ : first-order logic with a generalized quantifier $Q$ added (Mundici, 1985; Väänänen, 1999; Peters and Westerståhl, 2006). Modal perspectives make sense here, although there does not seem to be a full-fledged study of modal fragments of generalized quantifier logics. Coming at the same fragments from a different direction, modal logicians have added specific generalized quantifiers to the basic modal repertoire, such as counting quantifiers for numbers of successors in 'graded modal logic'. In the next section, we will encounter some formalisms that lie more in the latter vein of introducing quantifiers into modal logic.

It might have seemed that invariance and definability are the most obvious meeting point between modal neighborhood logic and generalized quantifier theory, but what we have mainly done is discussing obstacles. This may just be a sign of our not having found the key to unlocking the correct analogy - but at this stage, we are happy to hand over this challenge to the reader.

\footnotetext{
${ }^{15}$ Of course, this is a natural setting, witness the extensive literature on permutation invariance and logicality (e.g. Bonnay (2008), Feferman (1999), van Benthem (2019)).
} 
Coda. There are also possible comparisons of expressive power that do not run into the above considerations. For instance, generalized quantifier theory also has characterizations of special families of quantifiers through different combinations of properties. An example is the result in van Benthem (1986) that the classical quantifiers in the Square of Opposition are exactly those that have the three basic properties of Conservativity, Double Monotonicity (the quantifier allows for either upward or downward monotonicity inference in both of its arguments), and Variety (the quantifier is not constant in truth value on non-empty domains). This type of characterization does not seem to have been considered so far for capturing repertoires of modal operators.

\section{Modal logics of quantifiers}

Modal neighborhood semantics is used for very general purposes, where neighborhood structures can be topologies or more abstract algebraic structures validating only very weak logics close to the minimal logic of one or more algebraic operators added to Boolean algebra. But with neighborhood relations viewed as quantifiers, new concrete questions arise, that we will sample here. In what follows, for convenience, we restrict ourselves mostly to uniform neighborhood relations without any dependence on points. Also, we reverse our earlier bias, and concentrate mostly on the precise semantics for modal neighborhood logic.

\subsection{Modal logic of permutation-invariant quantifiers}

Much of modal logic is about axiomatizing reasoning with various modalities. Starting with the basic property of permutation invariance that makes quantifiers 'count', what sort of modal propositional logics arise when neighborhood relations are to be permutation invariant?

This task is related to completeness for standard logics with permutation invariant generalized quantifiers added. With uniform neighborhood relations, the modal setting is the special case of logics $P L(Q)$, that is, classical monadic predicate logic with just one unary generalized quantifier variable added (no identity, and no $\forall$ or $\exists$ ), whose interpretation $Q$ on a domain $W$ is such that

$$
\text { if } A \in Q,|A|=|B| \text { and }|W-A|=|W-B| \text {, then } B \in Q .{ }^{16}
$$

Richer logics with predicates of any arity have been investigated in Anapolitanos and Väänänen (1981), but in what follows, we look at our simpler case.

The minimal modal logic $\mathrm{E}$ (in the basic modal language) of arbitrary neighborhood frames just adds one inference rule to classical propositional logic: ${ }^{17}$

$$
\text { If } \vdash \varphi \leftrightarrow \psi \text {, then } \vdash \square \varphi \leftrightarrow \square \psi
$$

\footnotetext{
${ }^{16}$ With parametrized $N s, X$, the language becomes slightly richer, with point-dependent quantifiers. But as said above, we will ignore this extension here.

${ }^{17}$ That is, E is the set of formulas containing all classical tautologies, and closed under the rules of Uniform Substitution, Modus Ponens, and (RE), and this is exactly the set of formulas valid in all neighborhood frames; cf. Pacuit 2017.
} 
On uniform models, formulas of the form $\square \varphi$ or $\diamond \varphi$ are either true at all states or at none. Reduction principles like the following now become valid: ${ }^{18}$

$$
\begin{aligned}
& \square(p \wedge \square q) \leftrightarrow(\square q \wedge \square p) \vee(\neg \square q \wedge \square \perp) \\
& \square(p \vee \square q) \leftrightarrow(\square q \wedge \square \top) \vee(\neg \square q \wedge \square p) \\
& \square(\varphi \wedge \diamond \psi) \leftrightarrow(\square \neg \psi \wedge \square \perp) \vee(\diamond \psi \wedge \square \varphi) \\
& \square(\varphi \vee \diamond \psi) \leftrightarrow(\square \neg \psi \wedge \square \varphi) \vee(\diamond \psi \wedge \square \top)
\end{aligned}
$$

Let EU be E with (R1) - (R4) added. Using these, in EU all formulas are equivalent to formulas of modal depth $\leq 1$. The following result is folklore.

\section{Fact 8}

EU is the logic of all (finite) uniform neighborhood frames.

Now, what about validity when we require that the uniform neighborhood frames be permutation invariant? With the precise semantics, nothing happens.

\section{Fact 9}

Under the precise semantics, EU is the logic of all uniform and permutation invariant neighborhood frames.

Proof. Suppose $\forall_{\mathrm{EU}} \varphi$. By the above, there is a finite uniform model $\mathcal{M}=$ $(W, Q, V)$, with $W=\left\{s_{1}, \ldots, s_{n}\right\}$ and $\mathcal{M}, s_{j} \not \models \varphi$ for some $j$. Here $Q$ can be any subset of $P(W)$; we have no further information about its structure. What we need is a model where the quantifier is given by a set of numbers, where two sets of the same cardinality are either both in or both out. But there is a trick to achieve this. We assign numbers of objects to each state, standing for the number of points satisfying the corresponding state description, noting that the resulting numbers for disjoint unions are just the sums of those for the atoms inside. Here is the precise formulation:

\section{Lemma 10}

There is a function $f: \mathcal{P}(W) \rightarrow \mathbb{N}$ such that (a) $f(\emptyset)=0$, (b) $f$ is injective, and (c) $f$ is additive (i.e. if $X \cap Y=\emptyset$, then $f(X \cup Y)=f(X)+f(Y)) .{ }^{19}$

This can be proved by induction on $n$. The base case is trivial. If such an $f$ is given for $W=\left\{s_{1}, \ldots, s_{n}\right\}$, with $f(W)=N$, and $s$ is a new state, then for any new set $Y=\{s\} \cup X$, let $f^{\prime}(Y)=N+1+f(X)$, and $f^{\prime}(X)=f(X)$ for all old $X$. In particular $f^{\prime}(\{s\})=N+1$. Then $f^{\prime}$ is as desired.

Getting back to the proof of Fact 9, let $W^{\prime}$ be the union of pairwise disjoint sets $S_{1}, \ldots, S_{n}$, where $\left|S_{i}\right|=f\left(\left\{s_{i}\right\}\right)$. Let $\mathcal{M}^{\prime}=\left(W^{\prime}, Q^{\prime}, V^{\prime}\right)$, where $X \in Q^{\prime}$ iff for

\footnotetext{
${ }^{18}$ To see this, one can use the fact that the following schemes are valid on uniform frames: $\varphi[\square \psi] \leftrightarrow(\square \psi \wedge \varphi[\top]) \vee(\neg \square \psi \wedge \varphi[\perp])$ $\varphi[\diamond \psi] \leftrightarrow(\diamond \psi \wedge \varphi[\top]) \vee(\neg \diamond \psi \wedge \varphi[\perp])$
}

\footnotetext{
${ }^{19}$ This is really a fact about finite Boolean algebras: for any such algebra $\mathcal{A}$, there is an
} injective additive function $f$ from its domain to natural numbers, with $f(0)=0$. 
some $X \in Q,|Y|=f(X)$, and $V^{\prime}(p)=\bigcup_{s_{i} \in V(p)} S_{i}$. Finally, let $\rho: W^{\prime} \rightarrow W$ map each $s \in S_{i}$ to $s_{i}$. Then we have, for $s \in W^{\prime}$,

(a) $s \in V^{\prime}(p)$ iff $\rho(s) \in V(p)$

and, using the additivity and injectivity of $f$, for $X \subseteq W$,

(b) $\rho^{-1}[X] \in Q^{\prime}$ iff $X \in Q$

Together, (a) and (b) tell us that $\rho$ is a bounded morphism (in the neighborhoodsemantic sense, see Pacuit 2017) from $\mathcal{M}^{\prime}$ onto $\mathcal{M}$, from which it follows that if $s$ is such that $\rho(s)=s_{j}$, we have $\mathcal{M}^{\prime}, s \not=\varphi^{20}$

\subsection{Imposing more conditions}

Next, what happens if we impose further conditions on neighborhood relations, such as the upward monotonicity and intersection closure leading to the filter structure that is typical for standard modal logic over relational models? Indeed, the logic $\mathrm{K}$ is sound for an arbitrary neighborhood frame $(W, N)$ if and only if $(W, N)$ is a filter frame: that is, for each $s \in W$, the set $\{X \mid N s, X\}$ is a filter. In particular, when $W$ is finite, all filters are principal, and then the condition is exactly that $(W, N)$ is a relational frame. In this case, adding permutation invariance to uniformity changes the picture significantly:

\section{Fact 11}

The logic of finite uniform filter frames is K45. Adding permutation invariance changes the logic to being either S5 or the trivial logic $\mathrm{K} \square \perp$.

Proof. On any uniform neighborhood frame, in the presence of $\mathrm{K}$ the axioms (R1) $-(\mathrm{R} 4)$ are equivalent to $.4+.5$. For the converse we may, by the above, restrict attention to ordinary relational frames. If $\nvdash_{\kappa 45} \varphi$, there is, by the usual completeness theorem, a relational model $\mathcal{M}=(W, R, V)$ and $s \in W$ such that $R$ is transitive and euclidean and $\mathcal{M}, s \not \models \varphi$. Then $\mathcal{M}[s], s \not \forall \varphi$, with $\mathcal{M}[s]$ the rooted submodel of $\mathcal{M}$ generated by $s$. Since $R$ transitive and euclidean, it follows that $\mathcal{M}[s]$, seen as a neighborhood model, is in fact uniform.

For the second claim, note that there are only two permutation invariant principal filters on $\mathcal{P}(W)$ : either $\mathcal{P}(W)$ itself or $\{W\}$. The logic of the former is $\mathrm{K} \square \perp$, that of the latter (since accessibility is the universal relation) is $\mathrm{S} 5$.

But perhaps the most interesting question concerns the intermediate case of uniform frames $(W, Q)$ where $Q$ is only required to be closed under supersets. ${ }^{21}$ We can think of this as the logic of "enough", read as the existence of a set of witnesses of at least the threshold value associated with enough. This appears to be a harder case, and we only have some observations to offer.

\footnotetext{
${ }^{20}$ The style of proof in here is like that of the more general result in Anapolitanos and Väänänen (1981), and injective additive functions on Boolean algebras also occur in the analysis of the logic of comparative sizes in Ding et al. (2018).

${ }^{21}$ Rather than restricting to special neighborhood frames that are 'monotone' in this sense, we could also use the monotone semantics for the modal language over arbitrary frames.
} 
First, the minimal modal logic is now the system EM, which is like E except that the rule $(\mathrm{RE})$ has been replaced by

$$
\text { If } \vdash \varphi \rightarrow \psi \text {, then } \vdash \square \varphi \rightarrow \square \psi
$$

However, if permutation invariance is also required, we do get new validities:

\section{Fact 12}

If the propositional formula $\alpha$ is incompatible with $\beta$, and $\varphi$ with $\psi$, then

$$
\square(\alpha \vee \beta) \wedge \square(\varphi \vee \psi) \rightarrow \square(\alpha \vee \varphi) \vee \square(\beta \vee \psi)
$$

is valid for uniform monotone permutation invariant frames.

Remark: This principle is not valid without the monotonicity requirement. ${ }^{22}$

Proof. If the atomic symbols are $p_{1}, \ldots, p_{k}$, each formula can be seen as a disjunction of conjunctions of the form $p_{1}^{*} \wedge \ldots \wedge p_{k}^{*}$, where $p_{i}^{*}$ is $p_{i}$ or $\neg p_{i}$. List these conjunctions in some order, and name them $1, \ldots, 2^{k}$. Then each propositional formula can be identified with a set $A \subseteq\left\{1, \ldots, 2^{k}\right\}$. Now the above result will follow by repeated applications of the following

Claim Let $A_{1}, A_{2}$ be disjoint subsets of $\left\{1, \ldots, 2^{k}\right\}$. Take $n_{1} \in A_{1}, n_{2} \in A_{2}$, and let $\pi$ swap $n_{1}$ and $n_{2}$ but nothing else. Then:

$$
\models \square A_{1} \wedge \square A_{2} \rightarrow \square \pi\left(A_{1}\right) \vee \square \pi\left(A_{2}\right)
$$

Suppose that $\mathcal{M}=(W, Q, V)$ is permutation invariant and uniform, that $Q$ is monotone, and that $\mathcal{M} \models \square A_{1} \wedge \square A_{2}$ (or rather $\mathcal{M}, s=\square A_{1} \wedge \square A_{2}$, but, by uniformity, we can forget about $s$ for boxed formulas). So there are $X, Y \in$ $Q$ with $X=\llbracket A_{1} \rrbracket_{\mathcal{M}}$ and $Y=\llbracket A_{2} \rrbracket_{\mathcal{M}}$. By assumption, $X \cap Y=\emptyset$. Let $X_{j}=X \cap \llbracket\{j\} \rrbracket_{\mathcal{M}}$ and $Y_{j}=Y \cap \llbracket\{j\} \rrbracket_{\mathcal{M}}$, and let $x_{j}=\left|X_{j}\right|$ and $y_{j}=\left|Y_{j}\right|$, for $j=1, \ldots, 2^{k}$ ( $x_{j}$ and $y_{j}$ may be finite or infinite). Thus, $X=\bigcup_{j \in A_{1}} X_{j}$ and $Y=\bigcup_{j \in A_{2}} Y_{j}$. We need only distinguish two cases.

Case 1: $x_{n_{1}} \leq y_{n_{2}}$. Let $Z$ be like $X$, except that $X_{n_{1}}$ is replaced by a set $U$ of $x_{n_{1}}$ members of $Y_{n_{2}}$. It follows that $|Z|=|X|$, and $Z \subseteq \llbracket \pi\left(A_{1}\right) \rrbracket_{\mathcal{M}}$. Moreover, $|W-Z|=|W-X|$. (If $h$ is a permutation of $W$ which swaps $X_{n_{1}}$ and $U$ (but nothing else), then $Z=h(X)$.) By permutation invariance, $Z \in Q$, and so by monotonicity, $\mathcal{M}=\square \pi\left(A_{1}\right)$.

Case 2: $x_{n_{1}}>y_{n_{2}}$. Then we swap $Y_{n_{2}}$ with $y_{n_{2}}$ members of $X_{n_{1}}$, and similarly obtain $\mathcal{M} \models \square \pi\left(A_{2}\right)$. This proves the Claim and thereby our Fact.

However, so far, we have not been able to determine the complete modal logic for the monotone quantitative case.

Open problem: What is the complete modal logic of "enough"?

\footnotetext{
${ }^{22}$ Consider the case $\alpha=p \wedge q, \beta=p \wedge \neg q, \varphi=\neg p \wedge q, \psi=\neg p \wedge \neg q$. Then the antecedent of our formula is equivalent to $\square p \wedge \square \neg p$ and the consequent to $\square q \vee \square \neg q$, but this implication is clearly not valid if the quantifier $Q$ is not monotone: for example, let $|V(p)|=3,|V(q)|=|W|=5$, and let $Q=\{X \subseteq W:|X|=2$ or $|X|=3\}$.
} 
Remark. A natural addition to all of the above logics is a universal modality $U$, and hence an existential modality $E$. The resulting system corresponds to monadic predicate logics $P L^{+}(Q)$, where in addition to $Q$, we have the usual quantifiers $\forall$ and $\exists$ with their standard interpretation (though still no identity). In this setting, uniformity is expressible by a single formula:

a frame $(W, N)$ is uniform iff $\square \varphi \leftrightarrow U \square \varphi$ is true in that frame.

It is straightforward to give extensions of our earlier completeness results to this extended language, but we ignore details here.

\subsection{Modal logics of specific quantifiers}

Finally, instead of axiomatizing reasoning about classes of quantifiers, one can also look at the modal logic of specific cases. For a concrete example, consider the quantifier $P 1$ "precisely one". In the extended modal neighborhood language with an additional universal modality we have:

\section{Fact 13}

The complete modal logic of $P 1$ is axiomatized by $\mathrm{E}$ plus, for disjoint $\varphi, \psi$,

(a) $P 1(\varphi \vee \psi) \leftrightarrow(P 1 \varphi \wedge \neg E(\psi \wedge \neg \varphi)) \vee(P 1 \psi \wedge \neg E(\varphi \wedge \neg \psi))$

(b) $P 1 \varphi \rightarrow E \varphi$

Proof. It suffices to consider a consistent normal form of depth 1 , since these still exist for the present language with uniform quantifiers. Quantifiers P1 will only occur then in front of purely propositional formulas, that can be brought in disjunctive normal form.

Next, by repeated applications of the first axiom, we end up with a Boolean combination of cases (a) P1 attached to exactly one state description, (b) existential modalities over state descriptions, and (c) proposition letters. Bringing this Boolean combination in disjunctive normal form again, by the consistency, there must be at least one conjunction remaining, and such conjunctions consist of a state description, followed by a list of quantified statements of the form $(\neg) P 1 s d,(\neg) E s d$ for each state description $s d$. Here the second axiom makes sure there are no contradictions of the form $P 1 \varphi \wedge \neg E \varphi$.

Finally, it is easy to see by direct inspection that the conjunction can be satisfied semantically, reading off, for each state description, whether it needs to hold nowhere, in just one point, or in at least two points.

"Precisely one" is of course just one case ${ }^{23}$, similar logics can be written for any finite cardinality. For a logic of all finite cardinalities in a similar propositional language, cf. Pratt-Hartmann (2009) on the 'numerical syllogistic'.

Coda: Numerical transposition. Despite the modal propositional guise of the above logics, they can also be viewed numerically, as fragments of additive arithmetic. We briefly explain how this perspective works in our setting.

${ }^{23} \mathrm{~A}$ similar analysis would work for the binary quantifier "Precisely one $A$ is $B$ ". 
As an illustration, consider the earlier completeness of the neighborhood logic of an arbitrary permutation-invariant quantifier under the precise semantics. Now perform the following transposition on the satisfiability problem for a given modal formula in its normal form of modal depth 1:

Assign unique variables over natural numbers to each atomic state description, record the sums of these values that are to be in $Q$ according to the normal form as a finite list of additive terms $t_{1}, \ldots, t_{k}$, and those for the ones outside of $Q$ as a complement list $s_{1}, \ldots, s_{m}$. Next, to find a satisfying model for all of this, it suffices to solve this system of inequalities in the natural numbers: all numerical terms for the $s_{i}$ must have values different from all those for the $t_{j}$.

The reason for the adequacy of the equational procedure is this. If such values exist, then we can use them for the right multiplicities of the atoms, just as we did in the final part of the earlier modal completeness proof. ${ }^{24}$

Whether these systems of inequalities have a solution is decidable, via standard equation solving mechanisms. Indeed, looking at what is needed, this is only a small universal fragment of Presburger Arithmetic, which is decidable even in its full first-order version. Thus, another take on the topics in this section is that we are really axiomatizing small fragments of arithmetic in logical style. In particular, we conclude that all logics discussed here are decidable. ${ }^{25}$

\section{Conclusion}

In this exploratory paper, we have explored a suggested analogy between generalized quantifiers and modal neighborhood logics a bit further. We found interesting similarities in semantic notions of locality, new questions concerning modal logics for specific classes of quantifiers, and delicate but intriguing problems concerning the role of invariances in both realms.

Even so, we only scratched the surface, ignoring more general linguistic constructions such as polyadic or branching quantifiers and quantifier-like modal constructions, possible junctions between the model theory of generalized quantifier logics and modal logics, or possible counterparts in generalized quantifier theory for the rich theory of translations between modal logics.

One suggestive analogy ignored in this article concerns 'fine structure'. Much of modal logic is about semantic fine-structure, and the balance of expressive power and complexity in logic design. But equally well, generalized quantifier theory has sought to parametrize into easier and harder quantifiers, using definability in formal languages, or more computationally, devices such as 'semantic automata' (van Benthem, 1986)). Whether this congeniality can be made into a useful semantic interface remains to be seen. In addition to expressive fine-structure, however, there is also deductive fine-structure. Modal logics are

\footnotetext{
${ }^{24}$ If special conditions have to hold for the quantifier $Q$, such as monotonicity, then this can be worked into the method by adding suitable further inequalities closing off the $t$-list upward.

${ }^{25} \mathrm{~A}$ similar reduction to additive arithmetic applies to propositional logics with modalities for quantitative probabilities, cf. van Benthem (2017).
} 
often much simpler deductive subsystems of larger proof engines for first-order or higher-order logic. Generalized quantifier theory, too, has looked at deductive fine-structure of natural language, for instance, in the form of simple fast 'natural logics' inside more complex reasoning with quantifiers (cf. Moss, 2015). The two views of deductive fine-structure are not the same, and it would be of interest to export, for instance, natural logic thinking to the modal realm.

This paper is largely a set of pointers. Much remains to be done, but the glimpses we have shown may entice the reader to go further on the road ahead.

Acknowledgment We thank Nick Bezhanishvili, Larry Moss, Eric Pacuit, and an anonymous referee for helpful comments and useful information.

\section{References}

Anapolitanos, D. A. and Väänänen, J. (1981). Decidability of some logics with free quantifier variables. Zeitschrift f. math. Logic und Grundlagen d. Math., 27, 11-22.

Andréka, H., van Benthem, J., and Németi, I. (1998). Modal languages and bounded fragments of predicate logic. Journal of Philosophical Logic, 27(3), 217-274.

Andréka, H., van Benthem, J., and Németi, I. (2017). On a new semantics for firstorder predicate logic. Journal of Philosophical Logic, 46(3), 259-267.

Aucher, G., van Benthem, J., and Grossi, D. (2018). Modal logics of sabotage revisited. Journal of Logic and Computation, 28(2), 269-303.

Baltag, A. and Cinà, G. (2018). Bisimulation for conditional modalities. Studia Logica, 106:1, 1-33.

Barwise, J. and Cooper, R. (1981). Generalized quantifiers in natural language. Linguistics and Philosophy, 4, 159-219.

Blackburn, P., de Rijke, M., and Venema, Y. (2001). Modal Logic. Cambridge University Press, Cambridge.

Bonnay, D. (2008). Logicality and invariance. Bulletin of Symbolic Logic, 14:1, 29-68.

Bonnay, D. and Westerståhl, D. (2018). On the meaning of $\square$. Manuscript.

de Jongh, D. and Shirmohammadzadeh, F. (2018). Two neighborhood semantics for subintuitionistic logics. Tech report PP-2018-08, ILLC, University of Amsterdam.

Ding, Y., Harrison-Trainor, M., and Holliday, W. (2018). The logic of comparative cardinality. Manuscript, Logic Group, UC Berkeley.

Feferman, S. (1999). Logic, logics and logicism. Notre Dame Journal of Formal Logic, 40, 31-54.

Hansen, H. H., Kupke, C., and Pacuit, E. (2009). Neighbourhood structures: bisimilarity and basic model theory. In Logical Methods in Computer Science, volume 5 $(2: 2)$, pages $1-38$. 
Jacobs, B. (2016). Introduction to Coalgebra. Cambridge University Press, Cambridge.

Moss, L. (2015). Natural logic. In S. Lappin and C. Fox, editors, Handbook of Contemporary Semantic Theory, Second Edition, pages 646-681. Wiley-Blackwell.

Mundici, D. (1985). Other quantifiers: an overview. In J. Barwise and S. Feferman, editors, Model-theoretic Logics, pages 211-233. Springer-Verlag, Berlin.

Pacuit, E. (2017). Neighborhood Semantics for Modal Logic. Springer.

Pauly, M. (2001). Logic for Social Software. Ph.D. thesis, University of Amsterdam.

Peters, S. and Westerståhl, D. (2006). Quantifiers in Language and Logic. Oxford University Press, Oxford.

Pratt-Hartmann, I. (2009). No syllogisms for the numerical syllogistic. In O. Grumberg, M. Kaminski, S. Katz, and S. Wintner, editors, Languages: From Formal to Natural, pages 192-203. Springer.

Väänänen, J. (1999). Generalized quantifiers. In Generalized Quantifiers and Computation, LectureNotes in Computer Science, vol. 1754. Springer.

van Benthem, J. (1984). Foundations of conditional logic. Journal of Philosophical Logic, 13, 303-349.

van Benthem, J. (1986). Essays in Logical Semantics. North-Holland.

van Benthem, J. (1998). Program constructions that are safe for bisimulation. StudiaLogica, 60(2), 311-330.

van Benthem, J. (2002). Invariance and definability: two faces of logical constants. In W. Sieg, R. Sommer, and C. Talcott, editors, Reflections on the Foundations of Mathematics, pages 426-446. ASL Lecture Notes in Logic, 15.

van Benthem, J. (2011). Logical Dynamics of Information and Interaction. Cambridge University Press, Cambridge.

van Benthem, J. (2014). Logic in Games. The MIT Press.

van Benthem, J. (2017). Against all odds: When logic meets probability theory. In J. Katoen, R. Langerak, and A. Rensink, editors, Festschrift for Ed Brinksma, pages 239-253. Springer Lecture Notes in Computer Science 10500.

van Benthem, J. (2019). Semantic perspectives in logic. In G. Sagi and J. Wood, editors, The Semantic Conception of Logic. Cambridge University Press.

van Benthem, J. and Bezhanishvili, G. (2007). Modal logics of space. In M. A. et al, editor, Handbook of Spatial Logics, pages 217-298. Springer, Dordrecht.

van Benthem, J. and Blackburn, P. (2007). Modal logic: a semantic perspective. In J. van Benthem, P. Blackburn, and F. Wollter, editors, Handbook of Modal Logic, Studies in Logic and Practical Reasoning, pages 1-84. Elsevier.

van Benthem, J. and Bonnay, D. (2008). Modal logic and invariance. Journal of Applied Non-Classical Logics, 18(2-3), 153-173. 
van Benthem, J. and Pacuit, E. (2011). Dynamic logic of evidence-based beliefs. Studia Logica, 99(1), 61-92.

van Benthem, J., Bezhanishvili, N., and Enqvist, S. (2017). Instantial neighbourhood logic. The Review of Symbolic Logic, 10(1), 116-144.

Venema, Y. (2006). Algebras and coalgebras. In P. Blackburn, J. van Benthem, and F. Wolter, editors, Handbook of Modal Logic, pages 331-426. Elsevier Science, Amsterdam. 